\title{
ILLUSTRATIONS OF SYLOW'S THEOREMS ON GROUPS.
}

By Prof. Cayley.

The theorems 1, 2, and 3 in the paper Sylow "Théorèmes sur les groupes de Substitutions," Math. Ann. t. v. (1872), pp. 584-594 apply to groups in general, and not only to groups of Substitutions. They are as follows:

Theorem 1. If $n^{a}$ be the highest power of the prime number $n$ which divides the order of a group $G$, this group contains a group $g$ of the order $n^{a}:$ if, moreover, $n^{\alpha} v$ is the order of the highest group contained in $G$, the operations whereof are permutable with the group $g$, then the order of $G$ is of the form $n^{a} v(n k+1)$. [I write $k$ for Sylow's $p$, since it is convenient to have $p$ to denote a prime number, and for Sylow's "Substitutions" I write "Operations."]

Theorem 2. Everything being as in the preceding theorem, the group $G$ contains precisely $n k+1$ distinct groups of the order $n^{a}$, and these are obtained by transforming any one of them by the operations of $G$, each group being given by $n^{a} v$ distinct transformations.

Theorem 3 . If the order of a group is $n^{a}, n$ being prime, then any operation 9 whatever of the group may be expressed by the formula

$$
9=\theta_{0}^{i} \theta_{1}{ }_{1} \theta_{2}{ }^{l} \ldots \theta^{r}{ }_{a_{-1}} \text {, }
$$

where

$$
\begin{aligned}
& \theta_{0}^{n}=1, \\
& \theta_{1}^{n}=\theta_{0}^{a}, \\
& \theta_{2}^{n}=\theta_{0}^{b} \theta_{1}{ }^{c}, \\
& \theta_{3}^{n}=\theta_{0}^{d} \theta_{1} \theta_{2}{ }_{2}, \\
& \vdots
\end{aligned}
$$

and where

$$
\begin{aligned}
& \vartheta^{-1} \theta_{0} \vartheta=1, \\
& \vartheta^{-1} \theta_{1}{ }^{\vartheta}=\theta_{0}^{\beta} \theta_{1}, \\
& \vartheta^{-1} \theta_{2} \vartheta=\theta_{0}^{\gamma} \theta_{1}^{j} \theta_{2}, \\
& \vartheta^{-1} \theta_{3} \vartheta=\theta_{0}{ }^{6} \theta_{1}^{\zeta} \theta_{2}{ }^{n} \theta_{8} . \\
& \vdots
\end{aligned}
$$


But at present I attend only to the theorems 1 and 2 .

For instance, consider the group $G$ of the order $n=6$, $1, \beta, \beta^{2}, \alpha, \alpha \beta, \alpha \beta^{2}\left(\alpha^{2}=1, \beta^{3}=1, \alpha \beta^{2}=\beta \alpha, \alpha \beta=\beta^{2} \alpha\right)$. Here $n=2$ or $3:$ if $n=2$, we have $N=n^{\alpha} \nu(n k+1)=2.1(2+1)$; if $n=3$, we have $N=n^{a} v(n k+1)=3.2 .1$.

First, $n=2$; we should have a group $g$ of the order 2 ; one such group is $(1, \alpha)$, and the only group the substitutions whereof are permutable with $(1, \alpha)$ is the group $(1, \alpha)$ itself: for, taking any other operation of the group, for instance $\beta$, it is not true that $\beta(\gamma, \alpha)=(1, \alpha) \beta$, in fact the left-hand is $(\beta, \beta \alpha)$ and the right-hand is $(\beta, \alpha \beta)$ or $\left(\beta, \beta^{2} \alpha\right)$ : hence $n^{\alpha} v,=2 v,=2$, or $v$ is $=1$.

Hence, also by theorem 2, there should be 3 groups of the order 2 such as $(1, \alpha)$, viz. these are $(1, \alpha),(1, \alpha \beta),\left(1, \alpha \beta^{2}\right)$; derived from $(1, \alpha)$ as follows:

$$
\begin{array}{ll}
1(1, \alpha) 1^{-1} & =(1, \alpha), \\
\alpha(1, \alpha) \alpha^{-1} & =(1, \alpha), \\
\beta(1, \alpha) \beta^{-1} & =(1, \alpha \beta), \\
\beta^{2}(1, \alpha) \beta^{-2} & =\left(1, \alpha \beta^{2}\right), \\
\alpha \beta(1, \alpha)(\alpha \beta)^{-1} & =\left(1, \alpha \beta^{2}\right), \\
\alpha \beta^{2}(1, \alpha)\left(\alpha \beta^{2}\right)^{-2}=(1, \alpha \beta),
\end{array}
$$

$$
\begin{aligned}
& \text { since } \beta^{-1}=\beta^{2} \quad\left\{\begin{array}{l}
\text { and therefore } \\
\text { second term is }
\end{array}\right\} \quad \beta \alpha \beta^{2}=\alpha \beta^{2} \cdot \beta^{2}=\alpha \beta \text {, } \\
& " \beta^{-2}=\beta \quad " \quad " \beta^{2} \alpha \beta=\alpha \beta \cdot \beta=\alpha \beta^{2}, \\
& \text { " }(\alpha \beta)^{-1}=\alpha \beta \quad \text { " } \quad \alpha \beta \alpha \alpha \beta=\alpha \beta \cdot \beta=\alpha \beta^{2} \text {, } \\
& "\left(\alpha \beta^{2}\right)^{-1}=\alpha \beta^{2} \quad " \quad, \quad \alpha \beta^{2} \alpha \alpha \beta^{2}=\alpha \beta^{2} \cdot \beta^{2}=\alpha \beta,
\end{aligned}
$$

viz. the derivatives are $(1, \alpha),(1, \alpha \beta),\left(1, \alpha \beta^{2}\right)$, each twice.

Secondly, $n=3$, there should be here a group of the order 3 , viz. this is $\left(1, \beta, \beta^{2}\right)$. The group, the substitutions whereof are permutable with $\left(1, \beta, \beta^{3}\right)$ is the entire group $\left(1, \beta, \beta^{2}, \alpha, \alpha \beta, \alpha \beta^{2}\right)$; in fact, taking any substitution hereof, for instance $\alpha$, we have $\alpha\left(1, \beta, \beta^{2}\right)=\left(1, \beta, \beta^{2}\right) \alpha$, viz. the left-hand side is $\left(\alpha, \alpha \beta, \alpha \beta^{2}\right)$, and the right-hand side is $\left(\alpha, \beta \alpha, \beta^{2} \alpha\right),=\left(\alpha, \alpha \beta^{2}, \alpha \beta\right)$, which is the left-hand side, the change of order being immaterial; this is the meaning of the expression used, "the operations whereof are permutable with the group g." Hence, we have $n^{a} \nu,=3 v,=6$, or $\nu=2$; and thence, also $n k+1,=3 k+1,=1$, viz. $k=0$. There is thus only a single group of the order 3 , viz. the group $\left(1, \beta, \beta^{2}\right)$. 
As another instance I take the group of the order 12 formed by the positive substitutions of four letters, viz. these are

$$
\begin{array}{r}
1, a b . c d, \quad a b c, \\
a c . b d, \quad a c b \\
a d . b c, \quad a b d \\
a d b \\
a c d \\
a d c \\
b c d \\
b d c
\end{array}
$$

Here, taking $n=2$, we have $N=n^{a} v(n k+1)=2^{2} .3 .1$; there is a group $g$ of the order 4 , viz. this is

$$
(1, a b . c d, a c . b d, a d . b c),
$$

and the greatest group the substitutions whereof are permutable with this group $g$, is the entire group of the order 12 ; thus, considering any substitution thereof, for instance $a b c$, we have

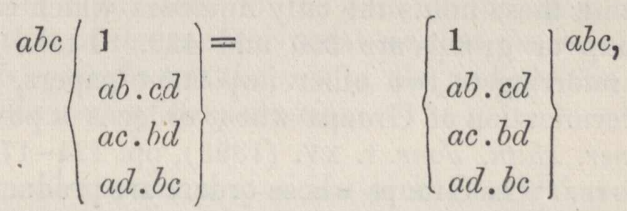

$$
\text { viz. left-hand is }\left(\begin{array}{l}
a b c \\
a c d \\
b d c \\
a d b
\end{array}\right) \text {, right-hand is }\left(\begin{array}{l}
a b c \\
b d c \\
a d b \\
a c d
\end{array}\right) \text {; }
$$

hence $n^{\alpha} v,=4 v,=12$ or $\nu=3$; whence also $n k+1,2 k+1,=1$ : and thus the foregoing group $g$ is the only group of the order 4.

Similarly taking $v=3$, we have $N=n^{a} v(n k+1),=3.1 .4$; there is a group $g$ of the order 3 , say $(1, a b c, a c b)$, the greatest group the substitutions whereof are permutable with $g$ is the group $g$ itself, viz. we have $n^{a} \nu,=3 \nu,=3$, or $\nu=1$; and then $n k+1,=3 k+1,=4$ : there are thus 4 groups of the order 3 , viz. these are

$$
(1, a b c, a c b),(1, a b d, a d b),(1, a c d, a d c),(1, b c d, b d c) .
$$


Reverting to the before-mentioned group of the order 6 , this not only contains each of the groups $(1, \alpha),(1, \alpha \beta)$, $\left(1, \alpha \beta^{2}\right)$ of order 2 , and the group $\left(1, \beta, \beta^{2}\right)$ of order 3 , but it is the permutable product of a group of order 2 into a group of order 3, say it is

$$
G=(1, \alpha)\left(1, \beta, \beta^{2}\right),=\left(1, \beta, \beta^{2}\right)(1, \alpha) .
$$

A group which is thus a permutable product of two factors is said to be a true product; and when it cannot be thus expressed as a permutable product of two factors it is prime or simple. A group, the order of which is equal to a prime number $p$ (the cyclical group of the order $p$ ) is simple, but the order may be a composite number and yet the group be simple-it was remarked by Galois (Liouville, t. XI. (1865), p. 409), that the order of the lowest simple group of composite order is $60,=2^{2} .3 .5$, and it has been recently shown, Holder "Die einfache Gruppen in ersten und Zweiten Hundert der Ordnungszahlen," Math. Ann. t. XL. (1892), pp. 55-88, that the only other composite order of a simple group in the first 200 numbers is 168 . Moreover, in the paper Cole "Simple groups from order 201 to order 500," Amer. Math. Jour. t. XIV. (1892), pp. 378-388, it is shown that within these limits the only numbers which can give a simple group or groups are 360 and 432 . I take the opportunity of referring to two other important papers, Young "On the determination of Groups whose order is a power of a prime," Amer. Math. Jour. t. Xv. (1893), pp. 124-178, and Cole and Glover "On Groups whose orders are products of three prime factors," pp. 191-220.

\section{COUNTER PEDALS.}

By $K$. Tsuruta, Tokio, Japan.

1. When a line is drawn from any point taken as origin parallel to the tangent at any point of a given curve, the locus of point of intersection of the line with the normal to the curve at that point is called the counter pedal of the curve with respect to the origin.*

* Craig, Amer, Jour. of Math. vol. Iv. p. 358. 